\title{
Is triglyceride to high-density lipoprotein cholesterol ratio a surrogates for insulin resistance in youth?
}

\author{
Antônio C. Oliveira ${ }^{1,2}$, Ana M. Oliveira ${ }^{2}$, Nelson Oliveira ${ }^{2}$, Atila Oliveira $^{3}$, Marcele Almeida ${ }^{1}$, \\ Lorena M. Veneza ${ }^{2}$, Ana Luisa Oliveira ${ }^{4}$, Luis Adan $^{5}$, Ana M. Ladeia ${ }^{1^{*}}$ \\ ${ }^{1}$ Bahian School of Medicine and Public Health, Science Development Foundation of Bahia, Salvador, Brazil; \\ *Corresponding Author: analadeia@uol.com.br \\ ${ }^{2}$ Department of Health, State University of Feira de Santana, Feira de Santana, Brazil \\ ${ }^{3}$ Science and Technology Faculty, Salvador, Brazil \\ ${ }^{4}$ Pontifical Catholic University, Campinas, Brazil \\ ${ }^{5}$ Department of Pediatrics, Federal University of Bahia School of Medicine, Salvador, Brazil
}

Received 16 November 2012; revised 16 December 2012; accepted 19 January 2013

\begin{abstract}
As elevated triglyceride (TG) to high-density lipoprotein cholesterol (HDL-C) ratio has been described as predictor for cardio metabolic disease with insulin resistance (IR) in the background, the aim was to assess the relationship between this ratio and IR in youth. Oral glucose tolerance test was performed in 318 Brazilian students (175 girls, $11.2 \pm 3.2$ years, BMI_z-score $1.2 \pm 1.4 \mathrm{SD}$ ). Measurements: BMI, waist circumference (WC), blood pressure (BP), lipids, insulin and high sensitivity $C$-reactive protein (hsCRP). Excessive weight and abdominal obesity were defined using BMI_z-score and WC and IR by Homeostasis model assessment-insulin resistance (HOMA-IR). TG/HDL-C median was 2.37. There was an association between TG/ HDL-C and WC, systolic and diastolic BP, insulin, HOMA-IR, hsCRP, $(p<0.001)$. Stratified TG/HDL$C$ into two groups, based on a cut off point of 2.73 (Group 1: $<2.73$ and Group 2: $\geq 2.73$ ). Adjusting for age, gender, ethnicity TG/HDL-C was an independent variable predicting IR $(P R=1.44$; Cl: $1.07-1.96 ; p=0.018)$. For every $1(\mathrm{~cm})$ in WC increased there was an addition of 0.04 of the value of TG/HDL-C and in those with TG/HDL-C $\geq$ 2.73 for every $10(\mathrm{~cm})$ increase in WC there was an improve of $4 \%$ in the ratio. In conclusion: In youth, the TG/HDL-C ratio could be a useful index for identifying groups with IR.
\end{abstract}

Keywords: Insulin Resistance; TG/HDL-C Ratio; Childhood Obesity; Metabolic Disease

\section{INTRODUCTION}

With recent increases in obesity among individuals in the pediatric age group, the rates of cardiovascular risk factors, are also rising $[1,2]$ with the background of insulin resistance (IR) $[3,4]$.

Classic markers explain only half the incidence of cardiovascular disease (CVD) $[5,6]$ thus the availability of relatively simple measures to identify subjects who are insulin resistant and have an atherogenic lipid profile linked to cardiometabolic disturbances would be of significant clinical benefit. Previous studies have demonstrated that fasting insulin and homeostasis model assessment (HOMA) are markers of IR [7] but there has been no attempt to standardize assays of insulin concentration to date. The triglyceride to high-density lipoprotein cholesterol (TG/HDL-C) ratio concentration has been reported to be closely related to IR in adults [8-11].

Hypertriglyceridemia is associated with low HDL-C, remnant-like particles, intermediate density lipoprotein and small, dense LDL-C, especially with the latter. This specific particle is an atherogenic lipoprotein with high capacity to promote oxidative modifications [12], but its requires highly sophisticated techniques to evaluate its size, and TG/HDL-C has been shown to be correlated to this specific size of LDL-C $[13,14]$.

It was demonstrated that youth with an elevated TG/ HDL-C are prone to express a proatherogenic lipid profile with atherogenic LDL particles $[15,16]$.

Therefore, to find commonly available marker that could help clinicians identify persons who had not only IR but also at heightened risk for atherogenic profile it was evaluated, in a sample of overweight Brazilian children and adolescents, the relationship between TG/HDL$\mathrm{C}$ ratio and abdominal obesity and IR.

\section{METHODOLOGY}

\subsection{Study Population and Study Protocol}

A multi-ethnic cohort study was conducted among 
overweight/obese youth recruited from public and private schools of Feira de Santana, Bahia, Brazil. Some of these participants are part of a larger study on the epidemiology of MS and IR in childhood obesity and thus have been reported on previously [17]. This sample comprised 318 healthy subjects (175 girls; 180 with excessive weight; mean age $\pm \mathrm{SD}, 11.2 \pm 3.2$ years).

The students attended the Unit initially to undergo a clinical and anthropometric examination after being instructed to refrain from vigorous physical activity on the day before the study date [17]. Blood pressure was measured in triplicate in accordance with the recommendations of the Task Force Report on High Blood Pressure in Children and Adolescents [18] and a standard 2-h oral glucose tolerance test (OGTT) was performed, in accordance with the American Diabetes Association guidelines [16,19]. Blood samples were withdrawn for the fasting measurement of glucose, insulin, HDL-C, TG and high sensitivity C-reactive protein (hsCRP) and following glucose ingestion, at 120 minutes, for measurement of plasma glucose. Sera were stored at $-70^{\circ} \mathrm{C}$ until they were analyzed. Plasma glucose, HDL-C, and TG concentrations were measured by automated enzymatic photometry; serum insulin levels by radioimmunoassay unit (Linco Laboratories) and hsCRP by fixed time nephewlometry [reporting range $0.2-10(\mathrm{mg} / \mathrm{L})$, coefficient of variation $<7 \%$ ) (Dade Behring, Deerfield, IL].

\subsection{Definitions}

To compare body mass index (BMI) across different ages, the BMI z-score was calculated, and a threshold of 1.5 or more defines excess weight for both genders. The degree of IR was defined using the HOMA-IR index, based on serum fasting glucose and insulin levels (product of glucose concentrations [expressed as milligrams per deciliter], and insulin [expressed as milliunits per milliliter] divided by a constant [405]) was used as a measure of IR and the cutoff used was 3.16 [20].

Subjects were classified into two groups based on a cut off point of 2.73 (Group 1: $<2.73$ and Group 2: $\geq$ 2.73).

As part of a protocol used by the authors [17], the WC was measured using the methodology described by the National Center of Health Statistics [21] and the blood pressure according to the Task Force on Blood Pressure Control in Children [18]. After training, a team formed by three nursery education students performed the anthropometric measurements in triplicate and the mean value was used. Ethical approval for the study was granted by the local Human Research Ethics Committee. Informed consent and assent were obtained from all guardians for the study, which was conducted in conformance with the 1975 Helsinki Declaration.

\subsection{Statistical Analyses}

To obtain cut-off values the overweight group was analyzed together with the obese group against the normal weight group. Receiver-operating characteristic (ROC) analysis was used to establish optimal cut-off values (Figure 1).

Data were presented as means \pm standard deviation (SD), except for the TG/HDL-C ratio, for which the median value was shown. Categorical variables were expressed as frequencies and proportions. The Chi-square $\left(\chi^{2}\right)$ or Fisher's exact tests were used whenever appropriate. The Student's- $t$ test (for symmetric variables) or Wilcoxon's rank-sum tests (for non-symmetric variables) were performed to compare continuous variables between groups above and below the cut-off value of the ratio. Spearman or Pearson correlation coefficients of the TG/HDL-C ratio, and clinical findings within biochemical parameters were presented. To control the confounding effects of age, sex, ethnic groups and BMI on the relationship between the TG/HDL-C ratio and IR, multiple logistic regression analyses were performed, and were used to model categorized TG/HDL-C ratio. The prevalence ratio (PR) was the measure of association used, confidence interval (CI) was reported and a p-value $<0.05$ was considered statistically significant.

SPSS (Statistical Packard for Social Sciences) for Windows statistical software version 17 was used for all calculations.

\section{RESULTS}

Based on the cut-off point of the ratio, the sample was classified in Group $1(\mathrm{n}=193)$, and Group $2(\mathrm{n}=125)$ and in Table 1, anthropometric, clinical and metabolic variables were presented. BMI and WC were significantly increased in the Group 2, as well as systolic and

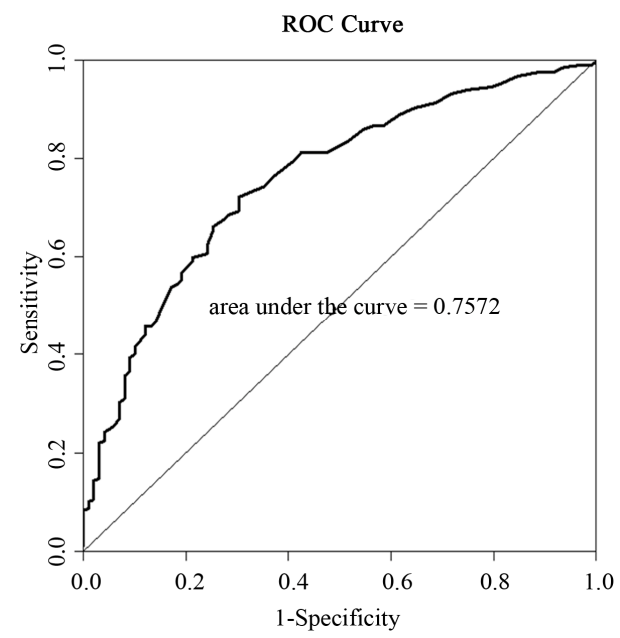

Figure 1. ROC curve for the association between TG/HDL-C ratio and waist circumference. 
Table 1. Baseline anthropometric. Clinical and metabolic characteristics of young Brazilians according to median TG/HDL-C ratio.

\begin{tabular}{|c|c|c|c|c|}
\hline Variables & $\begin{array}{c}\text { Sample } \\
(\mathrm{n}=318)\end{array}$ & $\begin{array}{c}\text { TG/HDL-C } \\
(<2.73) \\
(\mathrm{n}=193)\end{array}$ & $\begin{array}{c}\text { TG/HDL-C } \\
(\geq 2.73) \\
(\mathrm{n}=125)\end{array}$ & p value \\
\hline Age (y) & $11.2 \pm 3.2$ & $11.1 \pm 3.4$ & $11.3 \pm 2.9$ & 0.357 \\
\hline Gender (girls) & $175(55.0)$ & $121(69.1)$ & $54(30.3)$ & 0.001 \\
\hline \multirow{3}{*}{$\begin{array}{c}\text { Ethnic group } \\
\text { (white/mulatto/black) }\end{array}$} & $116(36.5)$ & $62(53.4)$ & $54(46.6)$ & \multirow{3}{*}{0.116} \\
\hline & 139 (43.7) & $92(66.2)$ & $47(33.8)$ & \\
\hline & $54(17.0)$ & $33(61.1)$ & $21(38.9)$ & \\
\hline BMI (z-score) & $1.2 \pm 1.4$ & $0.7 \pm 1.4$ & $1.7 \pm 0.8$ & 0.000 \\
\hline $\begin{array}{l}\text { Waist circumference } \\
\qquad(\mathrm{cm})\end{array}$ & $82.1 \pm 17.2$ & $77.3 \pm 16.2$ & $89.6 \pm 15.8$ & 0.000 \\
\hline $\begin{array}{l}\text { WC/stature ratio } \\
\text { (median) }\end{array}$ & $55.4 \pm 8.8$ & $0.3 \pm 0.4$ & $0.6 \pm 0.4$ & 0.000 \\
\hline $\mathrm{SBP}(\mathrm{mmHg})$ & $108.0 \pm 17.1$ & $103.9 \pm 15.6$ & $114.3 \pm 17.5$ & 0.000 \\
\hline DBP (mmHg) & $67.5 \pm 12.0$ & $65.8 \pm 11.6$ & $69.8 \pm 12.3$ & 0.005 \\
\hline $\begin{array}{l}\text { Fasting glucose } \\
\qquad(\mathrm{mg} / \mathrm{dL})\end{array}$ & $75.3 \pm 10.5$ & $74.9 \pm 10.8$ & $75.9 \pm 9.8$ & 0.329 \\
\hline $\begin{array}{l}\text { 2-h glucose } \\
(\mathrm{mg} / \mathrm{dL})\end{array}$ & $80.6 \pm 14.0$ & $79.2 \pm 13.9$ & $82.7 \pm 13.7$ & 0.026 \\
\hline $\mathrm{TC}(\mathrm{mg} / \mathrm{dL})$ & $175.8 \pm 33.0$ & $171.9 \pm 31.7$ & $181.7 \pm 33.8$ & 0.020 \\
\hline $\begin{array}{l}\text { LDL-cholesterol } \\
\text { (mg/dL) }\end{array}$ & $115.0 \pm 29.9$ & $114.0 \pm 28.0$ & $116.5 \pm 32.5$ & 0.734 \\
\hline $\begin{array}{l}\text { hsCRP }(\mathrm{mg} / \mathrm{L}) \\
\text { (median) }\end{array}$ & 2.0 & 1.4 & 3.0 & 0.012 \\
\hline $\begin{array}{l}\text { Fasting insulin } \\
\quad(\mu \mathrm{U} / \mathrm{mL})\end{array}$ & $17.3 \pm 14.6$ & $14.8 \pm 12.4$ & $21.2 \pm 16.7$ & 0.000 \\
\hline HOMA-IR & $3.2 \pm 2.8$ & $2.7 \pm 2.4$ & $4.0 \pm 3.2$ & 0.000 \\
\hline $\begin{array}{l}\mathrm{TC} / \mathrm{HDL}-\mathrm{C} \\
\text { ratio (median) }\end{array}$ & 4.4 & 4.0 & 5.2 & 0.000 \\
\hline $\begin{array}{l}\text { LDL-C/HDL-C } \\
\text { ratio (median) }\end{array}$ & 2.9 & 2.7 & 3.3 & 0.000 \\
\hline
\end{tabular}

TG/HDL-C: trygliceride to high-density lipoprotein cholesterol ratio; BMI: body mass index; SBP: systolic blood pressure; DBP: diastolic blood pressure; TC: total cholesterol; LDL-C: low-density lipoprotein cholesterol; HDL-C: high-density lipoprotein cholesterol; TG: Triglyceride; hsCRP: high sensitive C-reactive protein; HOMA-IR: homeostasis model assessment: insulin resistant; MS: Metabolic Syndrome.

Data are means \pm SE. $\mathrm{n}(\%)$ or medians. $\mathrm{p}^{\#}$ based on Wilcoxon's rank-sum test for continuous variables and $\chi^{2}$ test for dichotomous variables.

diastolic blood pressures. Positive associations were observed for TG/HDL-C ratio with WC $(\mathrm{r}=0.435 ; \mathrm{p}<$ $0.001), \mathrm{WC} /$ stature $(\mathrm{r}=0.388 ; \mathrm{p}<0.001)$, fasting insulin $(\mathrm{r}=0.321 ; \mathrm{p}<0.001)$, HOMA-IR $(\mathrm{r}=0.335 ; \mathrm{p}<0.001)$, systolic BP $(r=0.332 ; \mathrm{p}<0.001)$, diastolic $\mathrm{BP}(\mathrm{r}=0.185$; $\mathrm{p}=0.001)$, and hs-CRP $(\mathrm{r}=0.204 ; \mathrm{p}=0.001)$. Adjusting for age, gender, ethnicity TG/HDL-C ratio was an independent variable predicting IR $(\mathrm{PR}=1.44 ; \mathrm{CI}: 1.07$ $1.96 ; p=0.018)$.

For every $1-\mathrm{cm}$ increase in WC there were a improve of 0.04 in terms of unit of TG/HDL-C ratio and in those with TG/HDL-C above or equal 2.73 for every $10(\mathrm{~cm})$ increase in WC there were a improve of $4 \%$ in the ratio.

\section{DISCUSSION}

Others studies conducted by our group clearly showed that childhood obesity has high prevalence in developing countries [22] and leads to several cardio metabolic disturbances, such as increase in blood pressure [23,24] presence of fatty liver disease [25], IR [26], metabolic syndrome and subclinical inflammation [17]. Therefore, excessive weight is related to a preatherosclerosis status that starts early in life and is associated with increased mortality due to CVD [27]. The MS, is a constellation of interrelated metabolic risk factors that lead to the development of diabetes and CVD, and one of the most accepted underlying mechanisms for this syndrome is the disease of the adipose tissue with visceral adiposity and IR $[28,29]$ and this study clearly showed that the TG/ HDL-C ratio was associated with abdominal obesity and IR. Otherwise not all obese persons will develop IR, endothelial dysfunction, MS and cardiometabolic diseases, and paradoxically, a high number of non-obese individuals do suffer from these conditions [30]. A number of biomarkers described in literature, such as highmolecular weight subfraction of adiponectin, tumour necrosis factor alpha (TNF- $\alpha$ ), plasminogen activator inhibitor 1 (PAI-1), von Willebrand Factor (vWF), cytokines related to IR, inflammation, atherosclerosis, type 2 diabetes, dyslipidemia and hypertension [30-32] are not available for clinical practice. Furthermore the measurement of small low-LDL-C (phenotype B), which is associated with a fourfold atherogenic risk [33], or even insulin dosage that is closely related to IR are difficult to measure. As obesity and its correlated morbidities are world public health problems a simple marker for cardiovascular risk factor is needed for epidemiological surveys. Then, this study proposes for pediatric population the cutoff point of 2.73 rather than 3.5 normally used in adult patients [34]. This proposal is not arbitrary considering that children may have lower TG levels and higher HDL-C levels than adults. Thus, 2.73 would identify more children on IR state.

Lipid abnormalities have for long been related to atherosclerosis [35] but recently, lipid particle subfractions have also been implicated in the process [33].

The finding that an increased TG/HDL-C ratio is correlated with a higher HOMA index could indicate this ratio as a useful marker for identifying insulin resistant individuals. A study that had fasting measurement of plasma lipids and LDL particle concentrations and size showed a great ability of different TG/HDL ratios to identify overweight youth with atherogenic profile [16] adding to this marker more one reason to be used.

In conclusion we demonstrated that in overweight youth the TG/HDL-C ratio is associated with IR. These findings place obese youth with IR in the category of a group that needs especial attention with regard to identi- 
fication, prevention and treatment for cardiometabolic disorders. As an easily available marker, TG/HDL is a strong candidate for a surrogate index of IR and atherosclerosis. In children, a lower cutoff point should be established.

\section{DISCLOSURE}

The authors declare no conflict of interest and had full access to the data and take responsibility for its integrity. All authors have read and agree to the manuscript as written.

\section{ACKNOWLEDGEMENTS}

We are grateful to all the young persons who participated in the study and to the nursing students for the excellent care given to our subjects. This study was supported by the Research Foundation of Bahia (FAPESB), Bahia, Brazil. This paper is part of A.C.O's PhD. Thesis of the Bahian School of Medicine and Public Health Post Graduate Course.

\section{REFERENCES}

[1] Gardner, M., Gardner, D.W. and Sowers J.R. (2008) The cardiometabolic syndrome in the adolescent. Pediatric Endocrinology Reviews, 5, 964-968.

[2] Bereket, A. and Atay, Z. (2012) Current status of childhood obesity and its associated morbidities in Turkey. Journal of Clinical Research in Pediatric Endocrinology, 4, 1-7. doi: 10.4274 /jcrpe. 506

[3] Esteghamati, A., Khalilzadeh, O., Anvari, M., et al. (2008) Metabolic syndrome and insulin resistance significantly correlate with body mass index. Arch Med Res 39(8):803-808. doi:10.1016/j.arcmed.2008.08.004

[4] Burgert, T.S., Dziura, J., Yeckel, C., et al. (2006) Microalbuminuria in pediatric obesity: Prevalence and relation to other cardiovascular risk factors. International Journal of Obesity, 30, 273-280. doi:10.1038/sj.ijo.0803136

[5] Haffner, S.M. (2006) Relationship of metabolic risk factors and development of cardiovascular disease and diabetes. Obesity, 14, 121S-127S.

[6] Wilson, P., Agostinho, D., Levy, D., et al. (1998) Prediction of coronary heart disease using risk factor categories. Circulation, 97, 1837-1847. doi:10.1161/01.CIR.97.18.1837

[7] Bertoni, A.G., Wong, N.D., Shea, S., et al. (2007) Insulin resistance, metabolic syndrome, and subclinical atherosclerosis: The multiethnic study of atherosclerosis (MESA). Diabetes Care, 30, 2951-2956. doi: $10.2337 / \mathrm{dc} 07-1042$

[8] Kim, J.S., Kang, H.T., Shim, J.Y., et al. (2012) The association between the triglyceride to high-density lipoprotein cholesterol ratio with insulin resistance (HOMA-IR) in the general Korean population: Based on the national health and nutrition examination survey in 2007-2009. Diabetes Research and Clinical Practice, 97, 132-138. doi:10.1016/j.diabres.2012.04.022
[9] Kang, H.T., Yoon, J.H., Kim, J.Y., et al. (2011) The association between the ratio of triglyceride to HDL-C and insulin resistance according to waist circumference in a rural Korean population. Nutrition, Metabolism \& Cardiovascular Diseases, xx, 1-8.

[10] Giannini, C., Santoro, N., Caprio, S., et al. (2011) The triglyceride-to-HDL cholesterol ratio: Association with insulin resistance in obese youths of different ethnic backgrounds. Diabetes Care, 34, 1869-1874.

doi:10.2337/dc10-2234

[11] Quijada, Z., Paoli, M., Zerpa, Y., et al. (2008) The triglyceride/HDL-cholesterol ratio as a marker of cardiovascular risk in obese children; association with traditional and emergent risk factors. Pediatric Diabetes, 9, 464-471. doi:10.1111/j.1399-5448.2008.00406.x

[12] Krauss, R.M. (1994) Heterogeneity of plasma low-density lipoproteins and atherosclerosis risk. Current Opinion in Lipidology, 5, 339-349. doi:10.1097/00041433-199410000-00005

[13] Maruyama, C., Imamura, K. and Teramoto, T. (2003) Assessment of LDL particle Size by triglyceride/HDL-cholesterol ratio in non-diabetic, healthy subjects without prominent hyperlipidemia. Journal of Atherosclerosis and Thrombosis, 10, 186-191. doi:10.5551/jat.10.186

[14] Jia, L., Long, S., Fu, M., et al. (2006) Relationship between total cholesterol/high-density lipoprotein cholesterol ratio, triglyceride/high-density lipoprotein cholesterol ratio, and high-density lipoprotein subclass. Metabolism Clinical and Experimental, 55, 1141-1148.

doi:10.1016/j.metabol.2006.04.004

[15] Weiss, R., Otvos, J.D., Sinnreich, R., et al. (2011) The triglyceride to high-density lipoprotein-cholesterol ratio in adolescence and subsequent weight gain predict nuclear magnetic resonance-measured lipoprotein subclass in adulthood. Journal of Pediatrics, 158, 153-141. doi:10.1016/j.jpeds.2010.07.002

[16] Burns, S.F., Lee, S.J. and Arslanian, S.A. (2012) Surrogate lipid markers for small dense low-density lipoprotein particles in overweight youth. Journal of Pediatrics, in press.

[17] Oliveira, A.C., Oliveira, A.M., Almeida, M.S., et al. (2008) Alanine aminotransferase and high sensitivity Creactive protein: Correlates of cardiovascular risk factors in youth. Journal of Pediatrics, 152, 337-342. doi:10.1016/j.jpeds.2007.07.013

[18] National High Blood Pressure Education Program Working Group on Hypertension Control in Children and Adolescents (1996) Update on the 1987 Task Force Report on High Blood Pressure in Children and Adolescents: A working group report from the National High Blood Pressure Education Program. Pediatrics, 98, 649-658.

[19] American Diabetes Association (2006) Diagnosis and classification of diabetes mellitus. Diabetes Care, 29, S43-S48.

[20] Keskin, M., Kurtolgu, S., Kendirci, M., et al. (2005) Homeostasis model assessment is more reliable than the fasting glucose/insulin ratio and quantitative insulin sensitivity check index for assessing insulin resistance among obese children and adolescents. Pediatrics, 115, 500-503. 


\section{doi:10.1542/peds.2004-1921}

[21] (2008) National health and nutrition examination survey. Anthropometry procedures manual. http://www.cdc.gov/nchs/data/nhanes/bm.pdf.2002

[22] Oliveira, A.M., Oliveira, A.C., Almeida, M.S., et al. (2007) Influence of the family nucleus on obesity in children from northeastern Brazil: A cross-sectional study. BMC Public Health, 7, 235. doi:10.1186/1471-2458-7-235

[23] Oliveira, A.M.A., Oliveira, A.C., Almeida, M.S., et al. (2004) Environmental and anthropometric factors associated with infantile arterial hypertension. Arquivos Brasileiros de Endocrinologia \& Metabologia, 48, 849-854. doi:10.1590/S0004-27302004000600011

[24] Centers for Disease Control and Prevention (CDC) (2010) Prevalence of abnormal lipid levels among youthsUnited States, 1999-2006. Morbidity and Mortality Weekly Report, 59, 29-33.

[25] Oliveira, A.M., Oliveira, N., Reis, J.C., et al. (2009) Triglycerides and alanine aminotransferase as screening markers for suspected fatty liver disease in obese children and adolescents. Hormone Research, 71, 83-88. doi:10.1159/000183896

[26] Galli-Tsinopoulou, A., Kyrgios, I., Maggana, I., et al. (2011) Insulin resistance is associated with at least threefold increased risk for prothrombotic state in severely obese youngsters. European Journal of Pediatrics, 170, 879-886. doi:10.1007/s00431-010-1370-9

[27] Must, A., Jacques, P.F., Dallal, G.E., et al. (1992) Longterm morbidity and mortality of overweight adolescents. A follow-up of the Harvard growth study to 1922 to 1935 . The New England Journal of Medicine, 327, 1350-1355. doi:10.1056/NEJM199211053271904

[28] (2001) Executive Summary of the Third Report of the National Cholesterol Education Program (NCEP) Expert Panel on Detection, Evaluation, and Treatment of High
Blood Cholesterol in Adults (Adult Treatment Panel III). Journal of the American Medical Association, 285, 24862497.

[29] Oda, E. (2008) The metabolic syndrome as a concept of adipose tissue disease. Hypertension Research, 31, 12831291. doi:10.1291/hypres.31.1283

[30] Arita, Y., Kihara, S., Ouchi, N., et al. (1999) Paradoxal decrease of an adipose-specific protein, adiponectin, in obesity. Biochemical and Biophysical Research Communications, 257, 79-83. doi:10.1006/bbrc.1999.0255

[31] Mangge, H., Almer, G., Haj-Yahya, S., et al. (2008) Preatherosclerosis and adiponectin subfractions in obese adolescents. Obesity, 16, 2578-2584. doi:10.1038/oby.2008.439

[32] Giordano, P., Del Vecchio, G.C., Cecinati, V., et al. (2011) Metabolic, inflammatory, endothelial and haemostatic markers in a group of Italian obese children and adolescents. European Journal of Pediatrics, 170, 845-850. doi:10.1007/s00431-010-1356-7

[33] Bhalodkar, N.C., Blum, S., Enas, E.A., et al. (2006) Accuracy of the ratio of triglycerides to high-density lipoprotein cholesterol for predicting low-density lipoprotein cholesterol particle sizes, phenotype B, and particle concentrations among Asian Indians. American Journal of Cardiology, 97, 1007-1009. doi:10.1016/j.amjcard.2005.10.036

[34] Ostfeld, R., Mookherjee, D., Spinelli, M., et al. (2006) A triglyceride/high-density lipoprotein ratio $>$ or $=3.5$ is associated with an increased burden of coronary artery disease on cardiac catheterization. Journal of the CardioMetabolic Syndrome, 1, 13-15. doi:10.1111/j.0197-3118.2006.05323.x

[35] da Luz, P.L., Favarato, D., Faria-Neto Jr., J.R., et al. (2008) High ratio of triglycerides to HDL-cholesterol ratio predicts extensive coronary disease. Clinics, 63, 427-432. 